\title{
Nonlinear effects on Turing patterns: Time oscillations and chaos
}

\author{
J. L. Aragón, ${ }^{1}$ R. A. Barrio, ${ }^{2}$ T. E. Woolley, ${ }^{3,4}$ R. E. Baker, ${ }^{4}$ and P. K. Maini ${ }^{4,5}$ \\ ${ }^{1}$ Departamento de Nanotecnología, Centro de Física Aplicada y Tecnología Avanzada, Universidad Nacional Autónoma \\ de México, Apartado Postal 1-1010, Querétaro 76000, México \\ ${ }^{2}$ Departamento de Física Química, Instituto de Física, Universidad Nacional Autónoma de México, Apartado Postal 01000, \\ 76230 Distrito Federal, México \\ ${ }^{3}$ Oxford Centre for Collaborative Applied Mathematics, Mathematical Institute, University of Oxford, 24-29 St. Giles', \\ Oxford OX1 3LB, United Kingdom \\ ${ }^{4}$ Centre for Mathematical Biology, Mathematical Institute, University of Oxford, 24-29 St. Giles', \\ Oxford OXI 3LB, United Kingdom, \\ ${ }^{5}$ Oxford Centre for Integrative Systems Biology, Department of Biochemistry, University of Oxford, \\ South Parks Road OX1 3QU, United Kingdom
}

(Received 12 April 2012; published 8 August 2012)

\begin{abstract}
We show that a model reaction-diffusion system with two species in a monostable regime and over a large region of parameter space produces Turing patterns coexisting with a limit cycle which cannot be discerned from the linear analysis. As a consequence, the patterns oscillate in time. When varying a single parameter, a series of bifurcations leads to period doubling, quasiperiodic, and chaotic oscillations without modifying the underlying Turing pattern. A Ruelle-Takens-Newhouse route to chaos is identified. We also examine the Turing conditions for obtaining a diffusion-driven instability and show that the patterns obtained are not necessarily stationary for certain values of the diffusion coefficients. These results demonstrate the limitations of the linear analysis for reaction-diffusion systems.
\end{abstract}

DOI: 10.1103/PhysRevE.86.026201

PACS number(s): 05.45.Pq, 82.40.Bj, 87.18.Hf

\section{INTRODUCTION}

Reaction-diffusion systems can produce spatially periodic stationary patterns, usually known as Turing patterns. In his seminal work [1], Turing suggested the possibility of obtaining periodic patterns, oscillating both in time and space, and that they only occur with three or more morphogens. More recently, it has been shown that Turing patterns oscillating in time can be generated in a two-species system when Turing and Hopf instabilities interact [2], by coupling stationary Turing structures with oscillating layers [3], or in some bistable systems [4] in which neither Hopf nor wave instabilities are required.

Diffusion-induced chaos has been investigated due to its connection with chemical chaos [5] and to the existence of some biological phenomena displaying this behavior [6]. Spatiotemporal chaos in reaction-diffusion systems can arise in different ways, for instance from the interaction between Turing and Hopf bifurcations [7], by homoclinic explosions [8], by interaction with propagating fronts [9], or behind transition fronts simulated by moving boundaries [10].

In this paper we study a simple reaction-diffusion system with two species in a monostable regime and with a fixed domain size, which produces temporal oscillations and chaos in a region where linear analysis predicts only Turing patterns with no temporal oscillations. As a single parameter varies, the oscillating patterns undergo successive bifurcations, producing period doubling, tori, and chaos. Furthermore, when the ratio of diffusion coefficients fulfills the conditions for a Turing instability, we find the unexpected result that in a large region of parameter space, only Turing patterns oscillating in time emerge from the homogeneous steady state. The importance of these results is that they not only show the stringent limitations of linear analysis but also open up new perspectives on the application of reaction-diffusion systems to real biological and chemical systems.

\section{THE MODEL}

The reaction-diffusion model that we study in this work is the so-called Barrio-Varea-Aragon-Maini (BVAM) model [11]:

$$
\begin{aligned}
& \frac{\partial u}{\partial t}=D \nabla^{2} u+\eta\left(u+a v-C u v-u v^{2}\right), \\
& \frac{\partial v}{\partial t}=\nabla^{2} v+\eta\left(b v+H u+C u v+u v^{2}\right),
\end{aligned}
$$

with zero flux boundary conditions. This model presents a richness of behavior, making it suitable as a good "laboratory" to gain insight into the mechanisms of pattern formation [12]. We will keep parameters $D, C$, and $H$ free while the remaining parameter values will be $\eta=1, a=-1$, and $b=-3 / 2$. According to linear analysis, this choice ensures that the equilibrium point $(0,0)$, in the absence of diffusion, is a stable spiral for the values of $H$ considered in this paper and also that the trace of the Jacobian is negative as we shall see below.

\section{A. Linear analysis}

The diffusionless system has the following equilibrium points:

$$
\begin{aligned}
\left(u_{0}, v_{0}\right) & =(0,0), \\
\left(u_{\mp}, v_{\mp}\right) & =\left(\frac{-5 C \mp \sqrt{5} \sqrt{\Delta}}{4 H+4}, \frac{-5 C \mp \sqrt{5} \sqrt{\Delta}}{10}\right),
\end{aligned}
$$

where $\Delta=5 C^{2}-8 H+12$. We observe that if $\Delta>0$, then three real equilibrium points are present, but if $\Delta<0$, then 


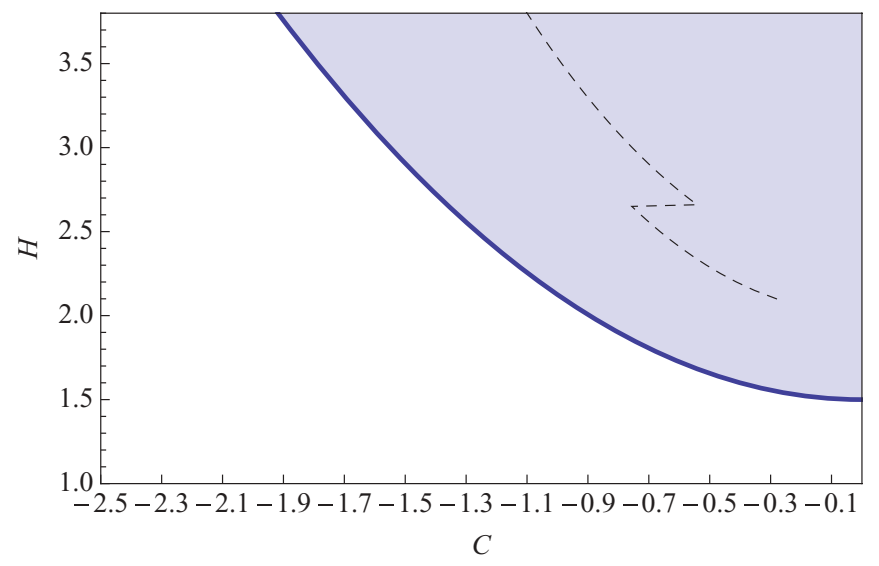

FIG. 1. (Color online) The bold line is the saddle-node bifurcation curve (2) of system (1) without diffusion. (Only the region $C<0$ is shown.) In the shaded region, $(0,0)$ is the only equilibrium point, which is linearly stable. The dashed curve is the locus of a Hopf bifurcation calculated numerically from Eq. (1) as described in Sec. III.

$(0,0)$ is the only real equilibrium point, hereafter denoted as $O$. The particular case $\Delta=0$ defines a saddle-node bifurcation curve given by the equation (see Fig. 1)

$$
H^{\mathrm{SN}}=\frac{1}{8}\left(5 C^{2}+12\right) \text {. }
$$

Along this curve, two equilibrium points are present, and to the left of $H^{\mathrm{SN}}$, the three equilibrium points are recovered. In the following we consider parameter values $C$ and $H$ inside the shaded region in Fig. 1. The Jacobian evaluated at $O$ is

$$
\mathbb{J}^{O}=\left(\begin{array}{ll}
1 & -1 \\
H & -\frac{3}{2}
\end{array}\right) .
$$

From this we obtain the eigenvalues $\lambda_{\mp}=\frac{1}{4}(-1 \mp$ $\sqrt{25-16 H}$ ) from which it is concluded that $O$ is linearly stable provided $H>3 / 2$. The value $H=3 / 2$ corresponds to the minimum of $H^{\mathrm{SN}}$. Thus, the shaded region bounded by $H^{\mathrm{SN}}$ in Fig. 1 corresponds to a monostable regime.

Since $\operatorname{tr}\left(\mathbb{J}^{0}\right)=-1 / 2$ is always negative, a Hopf bifurcation, which requires $\operatorname{tr}\left(\mathbb{J}^{0}\right)=0$, is not feasible.

\section{B. The Turing instability}

By considering diffusion in the linearized system, following the standard analysis for a Turing instability [13], we construct the matrix $\Gamma=\mathbb{J}^{0}-k^{2} \mathbb{D}$, where $\mathbb{D}=\operatorname{diag}(D, 1)$, from which we find $\operatorname{tr}(\Gamma)=-1 / 2-(1+D) k^{2}$. Since $D \geqslant 0$, it follows that $\operatorname{tr}(\Gamma)<0$; thus, neither oscillations with $k \neq 0$ (wave bifurcation) are allowed, and consequently, linear analysis predicts no oscillations at all. Since $O$ is linearly stable, there remains the possibility of having a Turing (diffusiondriven) instability. Using $D$ as the bifurcation parameter, the conditions for a Turing instability [13] yield the critical values

$$
\begin{gathered}
k_{c}^{2}=H-\frac{3}{2}+\sqrt{H\left(H-\frac{3}{2}\right)}, \\
D_{c}=\frac{8}{9}\left[H-\frac{3}{4}-\sqrt{H\left(H-\frac{3}{2}\right)}\right] .
\end{gathered}
$$

Notice that both critical quantities are independent of $C$.

\section{RESULTS}

The model in Eq. (1) was solved numerically in one dimension with zero flux boundary conditions and random initial conditions around the equilibrium point $(0,0)$. The second partial derivative terms in Eq. (1) are discretized using finite differences, including zero flux boundary conditions in the discretization. Using a mesh with $N$ nodes yields a set of $2 N$ ordinary differential equations which were solved using the software library CVODE [14] with a numerically calculated Jacobian matrix. Scalar absolute and relative tolerances of $10^{-9}$ were used, and $\Delta t=0.01$. We set $N=50$ and $\Delta x=$ 0.2 ; thus, the system size is $L=9.8$.

Since the critical parameters depend only on $H$, we first obtain a $D_{c}$ valid for the shaded region in Fig. 1, that is, the range $1.5<H<3.8$. In this interval $D_{c}$ decreases monotonically; thus, we need to consider only the values of $D_{c}$ when $H$ is evaluated at the boundaries of the inequality. From Eq. (4) we see that for $H=1.5, D_{c} \approx 0.66$ and that for $H=3.8, D_{c} \approx 0.083$. Thus, if we set $D=0.08$, Turing patterns should be produced in the range $1.5<H<3.8$. We now consider $C$ as a control parameter to explore its effect on the generated patterns. In what follows and unless otherwise indicated, we set $D=0.08$ and $H=3$. For this value of $H$, Eq. (3) gives $k_{c} \approx 1.9029$, and from the dispersion relation, it is found that the admissible (taking into account boundary conditions), most rapidly growing mode is $k=7 \pi / 9.8 \approx$ 2.244. Therefore, we expect a wavelength of $2 \pi / 2.244 \approx 2.8$ or $9.8 / 2.8 \approx 3.5$ stripes in the simulations.

In Fig. 2 numerical simulations for three different values of $C$ are displayed; in the left column, the space-time plots for $u$ up to 15000 time iterations only $(t=150)$ are shown for clarity. In the right column, the corresponding time series of the central point $u(25)$ are shown. As expected, for $C=-0.6$, as in Fig. 2(a), a stationary Turing pattern is obtained. When $C=-1$ [Fig. 2(b)], a stationary pattern is generated initially, but eventually regular oscillations emerge. If $C$ is decreased further, approaching the line $H^{\mathrm{SN}}$, the pattern oscillates in an apparently chaotic fashion as shown in Fig. 2(c) for $C=-1.5$. Since oscillations are not predicted by the linear analysis and the conditions for a Turing instability are valid for these parameter values (that is, there are admissible modes growing), it seems plausible to assume that these oscillations are a consequence of the nonlinear terms and, in particular, of the relative strength of the quadratic and cubic nonlinearities, given by the magnitude of $C$. Since an analytical treatment of nonlinearities is often prohibitive, we used the bifurcation software AUTO [15] to detect possible oscillatory instabilities.

In Fig. 3(a), we show the bifurcation diagram. As $C$ decreases from -0.1 , the stable stationary (Turing) state loses stability through a supercritical Hopf bifurcation at $C=-0.7546515$ (successive bifurcations will be discussed afterwards). Bifurcation diagrams can be generated for different values of $H$ so that a curve of Hopf bifurcations in a $C$ vs $H$ plane can be calculated numerically. By using steps of 0.01 in $H$, the resulting curve is plotted in Fig. 1 as a dashed line. This curve separates the monostability region where conditions for a Turing instability are satisfied into two regions: on the right-hand side of the Hopf curve, Turing patterns are realized, and on the left, patterns oscillate in time. 
(a)

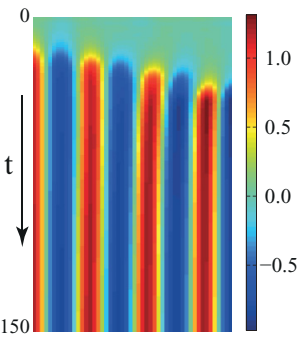

(b)

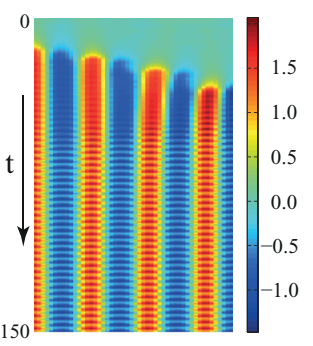

(c)

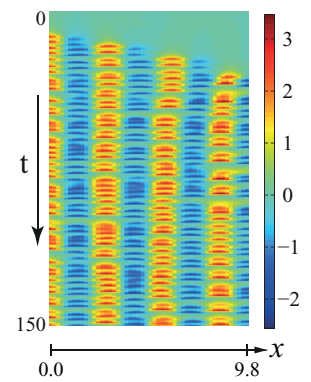

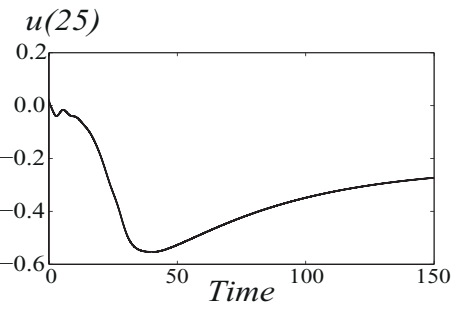
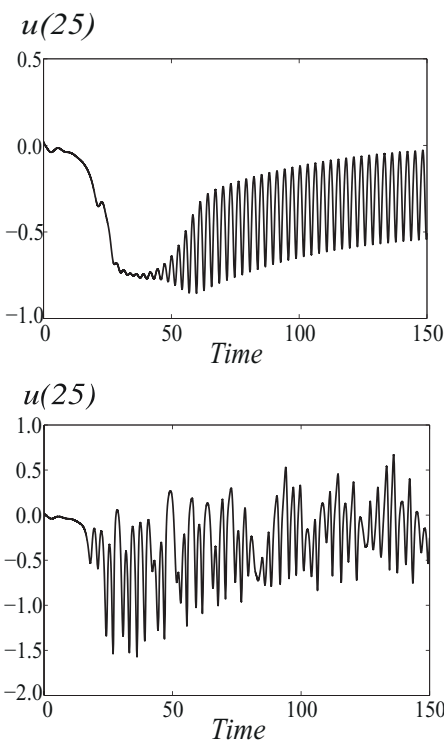

FIG. 2. (Color online) Numerical simulations of the system (1) in one dimension for $H=3$ and $D=0.08$. The left column shows the space-time plot of $u$ for three values of $C$. (The plots for $v$ are similar.) On the right, the time series registered at the central point $u(25)$ of the corresponding space-time plots are shown. (a) $C=-0.6$, (b) $C=-1$, and (c) $C=-1.5$.

It is worth mentioning that in the interval $2.1<H<2.5$, no Hopf bifurcation occurs, and in this region only oscillatory Turing patterns emerge from the homogeneous steady state instead of the expected stationary patterns. We observe from Eq. (4) that $D_{c}$ increases as $H$ diminishes such that when, for instance, $H=1.8$, the critical value $D_{c}=0.2801 \gg 0.08$. According to linear analysis, the uniform steady state becomes unstable to many modes, and one expects that the most rapidly
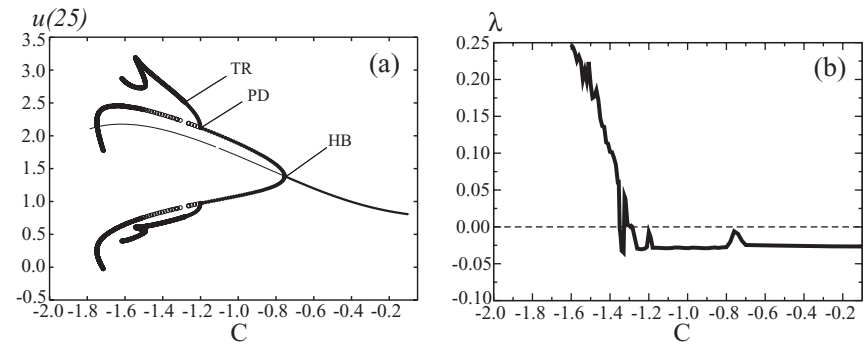

FIG. 3. (a) Numerically calculated bifurcation diagram of system (1) using AUTO. Supercritical Hopf (HB), period doubling (PD), and torus (TR) bifurcations are detected at $C=-0.7546515,-1.200656$, and -1.281831 , respectively. (b) Numerically calculated maximal Liapunov exponents.
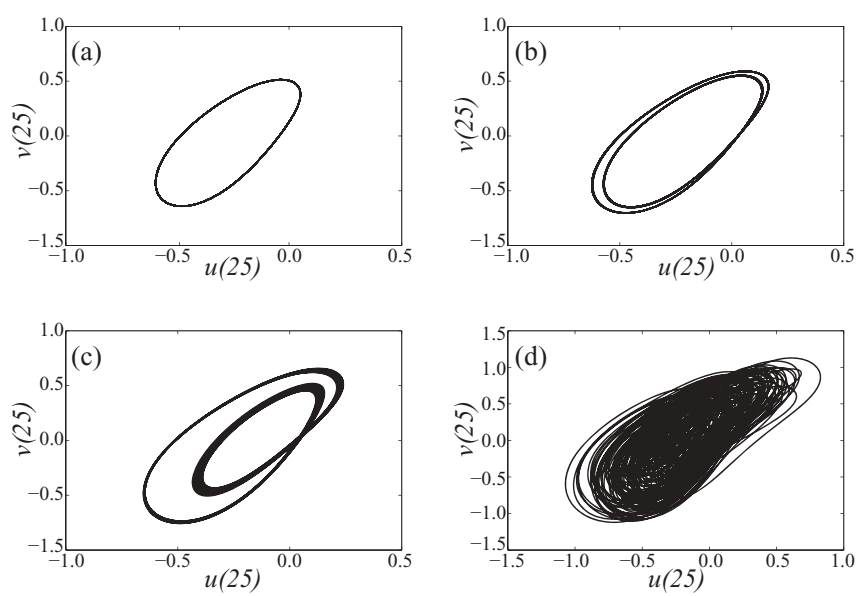

FIG. 4. Phase portraits of the central points for different values of $C$ after 50000 time iterations $(t=500)$. (a) $C=-1.15$, (b) $C=$ -1.21 , (c) $C=-1.285$, and (d) $C=-1.5$.

growing mode will eventually dominate. However, numerical simulations show that this is not so. If $D$ is just below 0.2801 , stationary Turing patterns are obtained as expected, but when $D \ll 0.2801$, only Turing patterns oscillating in time emerge from the homogeneous steady state.

\section{A. Chaos}

Once the Hopf bifurcation line is crossed, if $C$ decreases further, the oscillations become irregular, and near the $H^{\mathrm{SN}}$ line they appear to become chaotic. In Fig. 3(b), maximal Lyapunov exponents $(\lambda)$ are plotted versus $C$. According to our numerical results, $\lambda$ becomes zero at $C=-1.285$ and positive after this value except for a small region of stability $(-1.33 \leqslant C \leqslant-1.35)$. Consequently, chaos is expected when $-1.285<C<-1.33$ and when $C<-1.35$.

In Fig. 4, phase portraits are displayed for different values of $C$. After crossing the Hopf bifurcation line, a limit cycle is formed, as shown in Fig. 4(a), for $C=-1.15$. When $C=-1.21$, the cycle doubles its period as shown in Fig. 4(b). At smaller values of $C$ a torus is generated at $C=-1.285$ as in Fig. 4(c), and chaotic orbits, shown in Fig. 4(d), appear for $C=-1.5$. All these results are consistent with the successive bifurcations displayed in the bifurcation diagram of Fig. 3(a): after the Hopf bifurcation (at $C=-0.7546515$ ), the limit cycle loses stability through a period doubling for $C=-1.200656$, and this cycle itself loses stability through a torus at $C=-1.281831$.

The quasiperiodicity of the torus in Fig. 4(c) can be verified by calculating its power spectrum. In Fig. 5(a), the power spectrum for the torus $(C=-1.285)$ is shown. The limit cycle after the Hopf bifurcation has frequency $\omega_{1}=0.3382$, the period doubling bifurcation introduces the frequency $\omega_{1} / 2$, and the torus bifurcation introduces the new frequency $\omega_{2}=0.2732$. The ratio of frequencies $\omega_{1} / \omega_{2}=1.2379$ and, as it is impossible to discern numerically in this way whether or not a number is irrational, we cannot distinguish between quasiperiodic orbits or orbits with very long periods; thus, we verified that the Poincare plots are typical of quasiperiodic orbits (ringlike). The power spectrum for $C=-1.455$, shown 


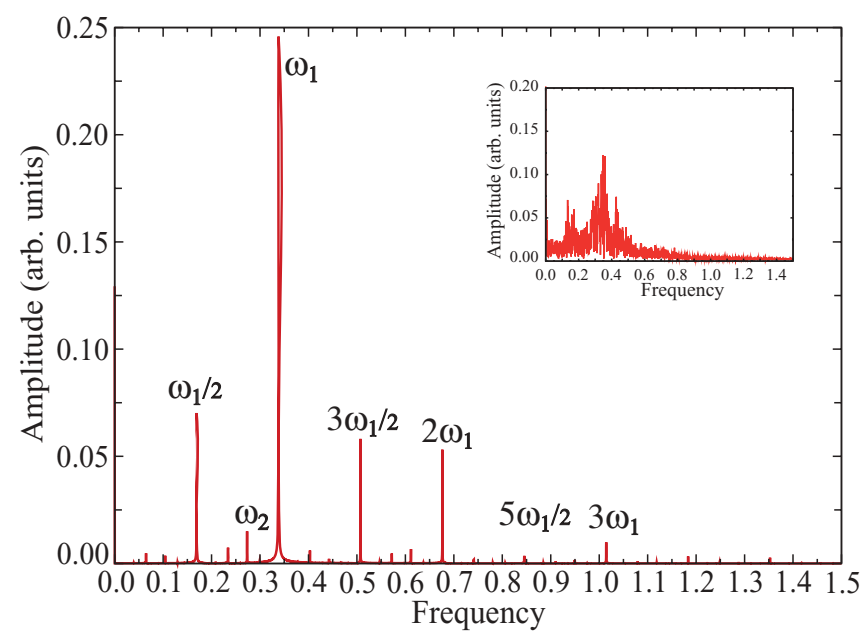

FIG. 5. (Color online) Power spectrum for $C=-1.285$ after 500000 iterations $(t=5000)$. The frequency $\omega_{1}=0.3382$ and its integer multiples appear after the Hopf bifurcation. The period doubling bifurcation introduces the frequency $\omega_{1} / 2$ and the torus bifurcation $\omega_{2}=0.2732$. Inset: Power spectrum for $C=-1.455$ showing a continuous band of frequencies, typical of a chaotic oscillation.

in the inset of Fig. 5, exhibits a continuous band of frequencies, confirming the chaotic nature of the oscillation.

From all of these numerical results, we observe the following sequence of bifurcations: stationary $\rightarrow$ Hopf $\rightarrow$ two-torus. Numerically, when a third frequency is about to appear (three-torus), small perturbations transform the quasiperiodic orbit into a chaotic orbit, or a strange attractor. This result is consistent with the well-known Ruelle-Takens-Newhouse route to chaos [16].

\section{B. Two-dimensional oscillating patterns}

In two dimensions, the parameter $C$ controls the type of Turing pattern, spots or stripes [11], so by varying this parameter, we can generate breathing spots or stripes in a
TABLE I. Parameter values used in supplementary movies.

\begin{tabular}{lccc}
\hline \hline Movie Name & $D$ & $H$ & $C$ \\
\hline D008H3C-1.mp4 & 0.08 & 3.0 & 1.0 \\
D008H3C-15.mp4 & 0.08 & 3.0 & 1.5 \\
D008H16C-02.mp4 & 0.08 & 1.6 & 0.2 \\
D008H35C-12.mp4 & 0.08 & 3.5 & 1.2 \\
\hline \hline
\end{tabular}

regular, quasiperiodic, or chaotic fashion. Some illustrative movies of the numerical simulations in two dimensions are included as supplementary material [17]. Equation (1) was implemented in the commercial finite element package COMSOL MULTIPHYSICS with zero flux boundary conditions and random initial conditions. The parameter values for the supplementary movies are shown in Table I.

\section{CONCLUSIONS}

In summary, we have studied a simple reaction-diffusion system that exhibits oscillations and chaos in a novel way. Turing patterns lose stability through a Hopf bifurcation which cannot be discerned from present linear analysis techniques. The bifurcation parameter $C$ is absent in the linear analysis, and as it varies, quasiperiodic and chaotic oscillations are generated in a large region of parameter space. A RuelleTakens-Newhouse route to chaos is identified. The main conclusion of this work is that linear analysis may be a stringent limitation for studying reaction-diffusion systems and that the nonlinearities play an important role, not only on stabilizing a pattern, but also in producing unsuspected bifurcations.

\section{ACKNOWLEDGMENTS}

This work was supported by CONACyT and DGAPAUNAM, México, under Grants No. 79641 and No. IN1003103 , respectively, and was based on work supported in part by Award No. KUK-C1-013-04, made by King Abdullah University of Science and Technology (KAUST).
[1] A. M. Turing, Philos. Trans. R. Soc., B 237, 37 (1952).

[2] L. Yang, M. Dolnik, A. M. Zhabotinsky, and I. R. Epstein, Phys. Rev. Lett. 88, 208303 (2002).

[3] L. Yang and I. R. Epstein, Phys. Rev. Lett. 90, 178303 (2003).

[4] V. K. Vanag and I. R. Epstein, Phys. Rev. E 71, 066212 (2005).

[5] Y. Kuramoto, Chemical Oscillations, Waves and Turbulence (Springer, Tokyo, 1984).

[6] L. F. Olsen and H. Degn, Q. Rev. Biophys. 18, 165 (1985).

[7] A. De Wit, G. Dewel, and P. Borckmans, Phys. Rev. E 48, R4191 (1993); V. Petrov, S. Metens, P. Borckmans, G. Dewel, and K. Showalter, Phys. Rev. Lett. 75, 2895 (1995).

[8] J. Elezgaray and A. Arneodo, Phys. Rev. Lett. 68, 714 (1992).

[9] J. H. Merkin, V. Petrov, S. K. Scott, and K. Showalter, Phys. Rev. Lett. 76, 546 (1996).

[10] J. A. Sherratt, Dynam. Stabil. Syst. 11, 303 (1996).

[11] R. A. Barrio, C. Varea, J. L. Aragón, and P. K. Maini, Bull. Math. Biol. 61, 483 (1999); T. Leppänen, Ph.D. thesis, Helsinki University of Technology, 2004 (unpublished).
[12] C. Varea, J. L. Aragón, and R. A. Barrio, Phys. Rev. E 60, 4588 (1999); D. A. Striegel and M. K. Hurdal, PLoS Comput. Biol. 5, e1000524 (2009); R. A. Barrio, R. E. Baker, B. Vaughan, K. Tribuzy, M. R. de Carvalho, R. Bassanezi, and P. K. Maini, Phys. Rev. E 79, 031908 (2009); T. E. Woolley, R. E. Baker, P. K. Maini, J. L. Aragón, and R. A. Barrio, ibid. 82, 051929 (2010).

[13] J. D. Murray, Mathematical Biology (Springer, Berlin, 2002), Vol. 2.

[14] S. D. Cohen and A. C. Hindmarsh, Comput. Phys. 10, 138 (1996).

[15] B. Ermentrout, Simulating, Analyzing, and Animating Dynamical Systems: A Guide to XPPAUT for Researchers and Students (Society for Industrial Mathematics, Philadelphia, 2002).

[16] S. Newhouse, D. Ruelle, and F. Takens, Commun. Math. Phys. 64, 35 (1978); J. P. Eckmann, Rev. Mod. Phys. 53, 643 (1981).

[17] See movies in the Supplemental Material at http://link.aps.org/ supplemental/10.1103/PhysRevE.86.026201 for numerical simulations of two-dimensional oscillating patterns. 